\title{
APRENDIZAJE SERVICIO, UNA ESTRATEGIA DE APRENDIZAJE SIGNIFICATIVO EN LA FORMACIÓN DE ESTUDIANTES DE TERAPIA OCUPACIONAL DE LA UNIVERSIDAD CENTRAL DE CHILE
}

\author{
SERVICE LEARNING, AS A MEANINGFUL LEARNING STRATEGY TRAINING STUDENTS \\ IN OCCUPATIONAL THERAPY AT THE CENTRAL UNIVERSITY OF CHILE
}

\section{Francisca Espinosa Torres $^{1}$ Viviana Rodríguez la Pietra. ${ }^{2}$}

\section{Resumen}

Los constantes cambios sociales, políticos y culturales, han generado problemáticas comunitarias que exigen de soluciones integrales e intersectoriales, efectivas y contextualizadas, dirigidas por profesionales competentes y comprometidos con el desarrollo social del país. Este artículo pretende dar a conocer una estrategia curricular necesarias para afrontar estos nuevos escenarios, los cuales han sido asumidos por la Educación superior a través del Proyecto Educativo y Planeación Estratégica de la Universidad Central de Chile y que permiten efectuar procesos de gestión a partir del conocimiento del contexto social y diagnóstico de las necesidades de la sociedad sobre la que se trabaja, construir proyectos educativos en los cuales se incorporen valores, principios de participación y compromiso social, además de utilizar metodologías pertinentes y acompañamiento académico adecuado y sostenible.

La escuela de Terapia Ocupacional de dicha Institución, asume el compromiso de llevar a cabo el desafío de incorporar la metodología de aprendizaje-servicio dentro de la formación de pregrado, reconociendo el impacto de la metodología en los procesos de aprendizajes significativos y su aporte al proyecto educativo desde el enfoque por competencias, experiencia que genera resultados significativos no sólo en términos de rendimiento, sino que además en los procesos de integración de conocimiento, movilización de recursos cognitivos y la interacción de estos frente a una problemática real, que dieron como resultado la elaboración de soluciones contextualizadas.

El análisis y sistematización de dichas experiencias pretenden evidenciar el impacto en los procesos de aprendizaje y compromiso que adoptan los estudiantes de pregrado, frente a vinculación, guiada y reflexiva, durante el desarrollo de una asignatura electiva, en la cual se ofrece un servicio comunitario, local y contextualizado bajo la Modalidad de Aprendizaje Servicio.

\section{Palabras claves}

Aprendizaje Servicio, Enfoque por competencias, Educación Superior, Terapia Ocupacional

1 Terapeuta Ocupacional. Magister en Terapia Ocupacional UNAB. Postítulo en Educación Superior de la Universidad Central de Chile. Académica Universidad Central de Chile, Miembro de la Red Nacional de Aprendizaje Servicio Chile (REASE). Lord Cochrane 417, Santiago, Chile. Francisca.espinosa@ucentral.cl

2 Terapeuta Ocupacional, Magister en Terapia Ocupacional. Académica de la Escuela de Terapia Ocupacional de la Universidad Central de Chile, Miembro de la Red Nacional de Aprendizaje Servicio Chile (REASE). Lord Cochrane 417, Santiago, Chile.viviana.rodriguez@ucentral.cl 


\begin{abstract}
:
The constant social, political and cultural changes, have produced community problems that demand a comprehensive, intersectoral, effective and contextualized solutions directed by competent and committed professionals to social development. This paper describes a necessary curriculum strategy to face these new situations, which have been assumed by the Higher Education through the Educational Project and Strategic Planning of the Central University of Chile, This allows for management processes from knowledge of the social context and diagnosis of local needs of society, also create educational projects in which values, principles of participation and social commitment are incorporated and use relevant methodologies and appropriate and sustainable academic support.
\end{abstract}

School of Occupational Therapy is committed to the challenge of incorporating service-learning methodology in undergraduate education, the impact of the methodology are recognizing significant learning processes and its contribution to the educational project from the skills approach, this experience has significant consequences not only in terms of academic performance, but also in the processes of integration of knowledge, cognitive mobilization of resources and the interaction of these facing a real problem, which resulted in the development of contextualized solutions.

The analysis and systematization of these experiences demonstrate the impact of learning processes and commitment to adopt undergraduates in the relationship, guided and reflective, during the development of an elective course, offering a community, specific service and contextualized from the Methodology Learning Service

\title{
Keywords
}

Service Learning, Competence approach, higher education, Occupational Therapy

\section{INTRODUCCIÓN}

Reconociendo a la educación como un proceso dinámico, complejo, multifactorial y multidimensional, que se va adquiriendo a través del análisis, e internalización de variada información, extraída del entorno (evidencia y experiencia) y que implica un proceso de aprendizaje, es que surge la necesidad de establecer un enfoque educativo que permita superar, de manera satisfactoria los desafíos que implica dicho proceso

Cómo se logra desarrollar una formación profesional completa, satisfactoria y atingente a las problemáticas socio-culturales que debe resolver el profesional hoy en día, en diferentes campos de acción y contextos según la disciplina elegida por los mismos, ha sido un cuestionamiento histórico, que pretende establecer ciertas definiciones de lo que se reconoce como Curriculum.

La multifactorialidad de las problemáticas sociohistóricas y socioculturales, ha generado la necesidad de contar con profesionales capacitados para enfrentar los desafíos actuales de manera competente.

Esta reflexión forma parte del proceso de perfeccionamiento y especialización en el campo de la docencia en educación superior y contribuye a la discusión que se realiza dentro de un marco formativo a quienes hemos optado por profundizar en la manera en cómo se va construyendo el conocimiento y cómo esperamos generar aprendizajes significativos, dirigidos a nuestros estudiantes y bajo una declaración de un enfoque basado en competencias dentro del Marco Institucional.

\section{Aprendizaje-Servicio una metodología para el aprendizaje significativo}

La metodología de Aprendizaje-Servicio, se ha formalizado como una metodología de enseñanza aprendizaje, a lo largo del mundo, tanto a nivel universitario como en el ámbito escolar. En algunos países fue promovida desde las políticas educativas, tales como Alemania, Argentina, Singapur o Estados Unidos (Tapia, 2010).

Para algunos autores esta estrategia es percibida como una propuesta pedagógica que permite desarrollar conocimientos y competencias a través de una práctica de servicio solidario. Una definición operacional más precisa es la introducida por Furco y Billig (citado por Tapia, 2010) al señalar que se trata de una "metodología pedagógica experiencial, que se puede 
definir como la integración de actividades de servicio a la comunidad en el currículum académico, donde los estudiantes utilizan los contenidos y herramientas académicas en atención a necesidades genuinas de una comunidad".

Según el Centro Latinoamericano de aprendizaje y servicio solidario, el eje de la metodología es la valorización de actividades académicas presentes en los programas de estudio, en una lógica de desarrollo y compromiso social, capaz de potenciar los aprendizajes formales desarrollados en aula. El conocimiento adquirido en el marco de la educación formal, se vuelca a la acción social, al servicio de necesidades de las comunidades y grupos sociales, contribuyendo a dar solución a un problema social específico y también a la profundización y aplicación de los conocimientos aprendidos (Tapia, 2010)

Una explicación más extensa nos plantean Puig; Batlle y Boch; Palos:

El aprendizaje-servicio se identifica con una actividad educativa que plantea la adquisición de conocimientos, conjuntamente con la aplicación de las materias de estudio y con la puesta en juego de actitudes y valores a través de la realización de un servicio pensado para cubrir las necesidades de la ciudadanía. Se trata de una propuesta educativa compleja e innovadora, que incluye muchos elementos ya conocidos, pero que aporta la novedad de vincular estrechamente el servicio y el aprendizaje en una sola actividad educativa articulada y coherente. (Citado por Lombardi, Giovanna y Lisett Range pp. 49)

Por otra parte es importante considerar que no cualquier tipo de experiencia o práctica educativa, constituye aprendizaje-servicio, ya que es primordial cumplir y garantizar estrechamente el aprendizajes rigurosos y simultáneamente las estrategias de acción social planeadas, las cuales deben impactar a una comunidad, los aprendizajes y vivencias de los estudiantes, y evaluar el impacto de sus resultados.

Así como la Universidad Central ha reconocido la metodología y la ha incorporado como una alternativa de propuesta educativa, diversas Universidades a nivel nacional la han introducido, reconociendo su compromiso y responsabilidad social universitaria. El compromiso docente y las disposiciones institucionales, son factores que contribuyen que la metodología este cada vez mas presente en distintas casas de estudio. Así mismo reconociendo su valor existe actualmente una Red Nacional de Aprendizaje y Servicio (REASE), la cual reúne a profesores, estudiantes, universidades, colegios, agrupaciones civiles y en general a todas las personas y organizaciones en Chile que estén interesadas en esta metodología. REASE (citado por Arratia, 2008) declara además que la responsabilidad social universitaria es "un elemento fundamental de la convivencia democrática, enfatizando la formación ética como persona; apoyando el proceso de crecimiento y de autoafirmación personal, y entregando espacios para la relación con otras personas, con su comunidad, su país y con el mundo"

\section{Reconstruyendo la Experiencia}

La escuela de Terapia Ocupacional, inicia su génesis en el año 2009 formando parte de la Facultad de Ciencias de la Salud de la Universidad Central de Chile. El programa de formación, respondiendo al sello que la distingue de "Inclusión Social y valoración de la diversidad desde un enfoque comunitario", y reconociendo el impacto de la metodología en los procesos de aprendizajes significativos y su aporte al proyecto educativo desde el enfoque por competencias, es que se decide incorporar una práctica en la asignatura Introducción a los Estudios Profesionales basada en la Metodología: Aprendizaje-Servicio que se materializa en una forma de diseñar, planificar y evaluar actividades de docencia, propias de la terapia ocupacional en la Comuna de El Monte, en la Provincia de Talagante de la Región Metropolitana de Chile. La incorporación de ésta práctica en el curriculum responde a la formación en el ámbito comunitario, para garantizar no sólo la adquisición de conocimientos teóricos y prácticos y el desarrollo de habilidades profesionales, sino también para la formación de Terapeutas Ocupacionales comprometidos con su contexto social. Todas las actividades planificadas dentro de la asignatura, se encuentran insertas en las acciones sociales impulsadas por un Centro Comunitario perteneciente a la Corporación Padre Patricio Espinosa, organización sin fines de lucro, cuya misión es trabajar con las personas más vulnerables de su territorio. En esta oportunidad estudiantes, junto a sus docentes, realizan actividades considerando las necesidades de los beneficiarios, tales como talleres de autocuidado para las personas mayores de la agrupación, actividades recreativas y educativas relacionadas con mejorar la calidad de vida de los adultos mayores de la comuna.

Esta metodología de Aprendizaje-servicio se replicó en la misma asignatura durante el año 2011, lográndose sistematizar la opinión de 90 estudiantes de Terapia 
Ocupacional, 3 docentes de Terapia Ocupacional y 3 líderes del Centro Comunitario, durante las prácticas realizadas el año 2010 y 2011. Los Instrumentos utilizados fueron encuestas y cuestionarios de auto-reporte de satisfacción.

Entre los resultados obtenidos se destaca la percepción de los estudiantes, quienes en un $98 \%$ se muestran satisfechos por la experiencia, dándole un calificativo de muy relevante y relevante el realizar una práctica en la Comunidad con grupos de personas vulnerables. Por otra parte los docentes reconocen la importancia de incluir esta experiencia tempranamente en la formación de pregrado y por último los líderes del Centro Comunitario otorgan una alta relevancia a las actividades realizadas por los estudiantes, considerando que el servicio otorgado impacta positivamente en sus condiciones de vida.

Las opiniones de los participantes del estudio mencionado, refieren un alto valor a la experiencia en la cual los contenidos académicos se integran con la problemáticas sociales de una comunidad. Importante es también el vínculo y relación establecidos entre estudiantes, docentes y destinatarios de modo de favorecer el aprendizaje mutuo de manera colaborativa. Esta experiencia contribuye a reconocer una nueva área de desarrollo de la profesión, hasta ahora poco explorada por los estudiantes, ya que en el colectivo social han predominado las áreas de trabajo disciplinar en el ámbito biomédico o de rehabilitación tradicional. Se ha logrado detectar, con esta experiencia, que la práctica comunitaria logra ofrecer mayores oportunidades de desarrollo profesional, consolidar la decisión vocacional de los estudiantes, reconocer la importancia de la profesión en ámbitos de acción donde aún no se instaura, además de favorecer la motivación por continuar desarrollando actividades solidarias en la comunidad, reforzando así los valores de responsabilidad social. Las docentes por su parte valoran el aporte que la profesión puede realizar a grupos vulnerables, que presentan restricciones en el acceso a servicios de Terapia Ocupa- cional. De igual manera esta estrategia de aprendizaje contribuye al descubrimiento de la profesión por parte de los destinatarios del servicio otorgando en esta experiencia una oportunidad de acercarse y empoderarse de recursos cognitivos y herramientas prácticas que les permitan analizar y resolver temas que les son sentidos, como el cuidado del adulto mayor, prevención de la discapacidad y cuidado de la salud mental.

Esta experiencia permite destacar como la metodología de Aprendizaje- Servicio brinda prácticas pertinentes que permiten relacionar el aprendizaje significativo con las experiencias contextualizadas. Las prácticas profesionales de los estudiantes del primer semestre de la carrera desarrolladas en un contexto comunitario socialmente vulnerable enriquecieron la formación profesional disciplinaria, al igual que los procesos de sensibilización y concientización de problemáticas colectivas y compromiso social. Los estudiantes lograron desarrollar un aprendizaje basado en el trabajo comunitario, destacando la importancia de contar con el compromiso de los diversos actores involucrados, es decir, estudiantes, académicos y comunidad, siendo relevante el acercamiento y conocimiento de las dinámicas de los diferentes protagonistas, identificando los elementos contextuales reales de una comunidad con múltiples necesidades, relacionadas con el nivel pobreza y vulnerabilidad social en el que se encuentran.

Reconociendo el impacto de la metodología y su aporte al proyecto educativo, la escuela decide incorporar a la malla de formación un electivo de formación general a contar del año 2012 hasta la actualidad, en los cuales los estudiantes realizan actividades prácticas en diversos centros e instituciones que benefician a personas en situación de vulnerabilidad y exclusión social. A modo de síntesis se presentan algunos ejemplos de dichos proyectos desarrollados durante el año 2013-2014 en la Escuela de Terapia Ocupacional de la Universidad Central de Chile. 
A) Proyecto 1: “Un juego de mesa herramienta de apoyo para niños con cáncer, residentes en Casa de Acogida de la Fundación nuestros Hijos, en Santiago de Chile": El proyecto consiste en la elaboración de un juego de mesa, el que cumple la función de facilitar la reflexión y toma de conciencia de quienes interactúan a través de él. El juego de mesa contemplando las características de los niños con cáncer, tanto a nivel de desarrollo psicomotor, como también que sus materiales puedan ser higienizados o lavados con facilidad. Su diseño considera la necesidad de los participantes en cuanto a conocer aspectos relacionados con diversas actividades cotidianas del hogar y las precauciones que se deben tener en cuanto a hábitos y rutinas de higiene. Se propone que el juego pueda ser utilizado con niños y sus padres, quienes también son beneficiarios del proyecto.

B) Proyecto 2: "Taller de Yoga y Juego como herramienta para promover el desarrollo psicomotor en los niños de la Casa de Acogida, Grada" Las estudiantes semanalmente asisten a Grada y realizan actividades lúdicas y sesiones de Yoga, contando con la participan activa niños y educadoras.

La planificación y orientación de las actividades contempla las necesidades de los niños, la cuales están principalmente enmarcadas en la estimulación del juego acordes a su edad y en habilidades cognitivas. El proceso tuvo una duración de 12 sesiones, culminando el proyecto con la capacitación a las educadoras en cuanto a las estrategias de juego y Yoga.

C) Proyecto 3: "Taller de Desarrollo personal y participación social para hombres en situación de calle, que asisten a la Hospedería Padre Lavín, del Hogar de Cristo en Santiago." En esta oportunidad el equipo de estudiantes realiza un Taller grupal, orientado al desarrollo de habilidades personales de los beneficiarios, esta estrategia se diseña posterior al diagnóstico participativo realizado con los beneficiarios directos, quienes problematizan esta necesidad. El proyecto tiene una duración de once sesiones de talleres efectuados bajo una metodología activa, práctica y vivenciales, desarrolladas a través de dinámicas grupales y análisis reflexivo de las vivencias, donde los participantes abordan las diversas habilidades personales, necesarias para la sobrellevar la vida cotidiana

Los resultados fueron igualmente significativos tanto para estudiantes, docentes y usuarios, según lo identificado en diversos instrumentos de registro utilizados a lo largo del proceso y al finalizar el periodo académico de la asignatura. Durante el año 2013 y 2014 el Programa de Desarrollo Social, perteneciente a la Vicerrectoría Académica, entrega un fuerte respaldo, tanto a la Escuela de Terapia Ocupacional, como a los docentes que implementaron la metodología, reconociendo la importancia y el valor de ésta para la formación profesional, promoviendo el uso de la metodología de aprendizaje-servicio a través de diferentes instancias de perfeccionamiento docente, apoyo financiero a proyectos y apoyo en la difusión hacia otras Escuelas sobre el impacto logrado.

La coherencia de la metodología considerando sus principios y valores, sumada a la visión de la Escuela de Terapia Ocupacional manifestada en la declaración del Perfil de Egreso, el cual considera una serie de Dominios y competencias relacionadas con un fuerte compromiso social y pensamiento crítico, cercanos y reflexivos ante las necesidades de la sociedad, facilitaron que esta metodología sea hoy reconocida y valorada por el cuerpo docente y por los estudiantes de nuestra escuela.

La metodología de Aprendizaje-servicio incorporada ya como estrategia pedagógica en una Asignatura Electiva de Formación de nuestra carrera, se orienta a que los estudiantes desarrollen proyectos comunitarios, para ello se contempla un proceso guiado por las etapas convencionales en el ciclo de diseño y evaluación de proyectos sociales, esto es: diagnóstico, planificación, ejecución y cierre. En el marco de esta experiencia de aprendizaje, los estudiantes consiguen aplicar los contenidos de la asignatura y transferir aprendizajes adquiridos en su formación, para construir, participativamente con socios comunitarios, un proyecto con pertinencia social y de carácter académico. En términos operativos, los estudiantes divididos en grupos de trabajo se vinculan durante todo un semestre con un socio 
comunitario específico. Podemos entender este proceso de servicio como

"Actividades complejas que requieren la sistematización de objetivos y tareas tanto de servicio como de aprendizaje, que se engranan en un proyecto bien articulado que se ejecuta en diferentes fases y que fomentan una mirada crítica y reflexiva respecto a retos socioculturales y medioambientales con la finalidad de mejorarla a través de la participación y el compromiso cívico. (López, Peña \& Hernández, 2014, p.506)

La asignatura además cuenta con la particularidad de docentes guías (articulados por un docente coordinador), que supervisan y acompañan el trabajo de cada uno de los grupos desde el contacto inicial con el socio comunitario hasta la devolución participativa de resultados.

Cabe destacar que una de las principales herramientas incorporadas en la metodología es la reflexión del estudiante, ante esto Torres (2004) indica que "Es a través de la reflexión constante y profunda sobre el quehacer práctico y su relación con los contenidos académicos que el servicio pasa a ser una experiencia de aprendizaje significativo" (p.30). Al inicio del desarrollo de la asignatura y previo al desarrollo del proyecto de Aprendizaje-Servicio en la comunidad, los estudiantes realizan una primera aproximación a través de una reflexión dirigida por el docente, cuyo objetivo es analizar y profundizar distintas realidades sociales de nuestro país, sus determinantes y el aporte que puede otorgar desde la mirada de Terapia Ocupacional. La actividad dirigida consiste en una revisión bibliográfica individual, cuya información recopilada se comparte en una mesa de discusión en la sala de clases, en donde participan los docentes guías. Este espacio de reflexión anticipa a los estudiantes y fortalece su rol dentro de los proyectos de aprendizaje-servicio. Un segundo espacio de reflexión formal, tiene como objetivo analizar la experiencia vivida en el proceso de Aprendizaje-servicio. Es una instancia de conversación con el equipo de estudiantes, guiada por el docente coordinador durante el proceso de implementación del proyecto. Esta instancia busca el análisis y reflexión de los estudiantes en cuanto a los aprendizajes adquiridos y al servicio realizado, junto con esto se solicita a los estudiantes un ensayo individual, en el que deben desarrollar algunos de los aspectos destacados de la reflexión. El tercer momento de reflexión tiene como fin analizar las fortalezas y debilidades del proceso de Aprendizaje-servicio, se realiza al finalizar el proceso de implementación del proyecto, a través de un espacio grupal en aula, en él los distintos grupos dialogan y problematizan en torno a lo vivido.
Por último, es importante destacar la participación de los docentes guías en el proceso de intervención-acción y su aporte a la reflexión permanente, siendo clave este acompañamiento para guiar las decisiones o modificaciones del proyecto y así favorecer el aprendizaje profundo desde las vivencias y los aspectos técnicos del servicio ofrecido.

\section{Vinculación de la experiencia con aspectos teóricos y bioéticos}

La Terapia Ocupacional como disciplina, desde sus inicios declara valores y principios integradores tales como la dignidad humana y el punto de vista holístico, reconociendo la conexión entre mente y cuerpo, considerando también a la persona en unión con el medio en el que vive, a través de la participación en ocupaciones (Kielhofner,2004).

A lo largo de la historia de nuestra disciplina, el rol profesional se ha visto marcado por intervenciones ligadas directamente con personas que viven situaciones de exclusión, vulnerabilidad e injusticia social. Nuestra historicidad demanda nuevos desafíos para la práctica profesional, dejando de lado aquellas intervenciones cuyo foco es la patología o déficit, centradas en acciones que buscan normalizar o adaptar a las personas a una sociedad. Estas prácticas han resultado insuficientes para enfrentar las necesidades sociales actuales, entendiendo que los problemas sociales, que dan como resultado la desigualdad y vulnerabilidad, son producto de las relaciones sociales típicas de la acumulación capitalista. En este sentido desde una perspectiva crítica, el Terapeuta Ocupacional debe generar procesos de concientización y acción de forma articulada, buscar la emancipación de las personas, de manera que garantice el respeto a la diversidad y a la justicia social. Así la emancipación implica la consciencia del derecho de tener derechos (Galheigo, 2012).

Los aspectos mencionados anteriormente refuerzan la necesidad de mirar los lineamientos que guían la formación de profesionales de la salud, especialmente Terapeutas Ocupacionales que orientan su labor profesional hacia la multidimencionalidad tanto de problemáticas como de estrategias de intervención, acotadas a un contexto en particular y desde una mirada integral. El contexto histórico, como hemos mencionado anteriormente evidencia la necesidad de un cambio social, reflexión que viene haciendo eco desde hace algunos años, tanto a nivel nacional como internacional. 
Bajo este escenario es importante destacar el Congreso Mundial de Terapeutas Ocupacionales del año 2010, el cual significó un enorme empuje para reflexionar sobre nuestras prácticas y su perspectiva social.

Es en este sentido que la educación superior, ha adquirido una relevancia fundamental para quienes creemos en la transformación de nuestra sociedad, en una sociedad más digna, inclusiva, respetuosa, cohesionada y equitativa. Bajo esta reflexión se considera que la formación universitaria es un pilar primordial para generar estos cambios, sin embargo es fundamental el aporte de los diversos actores comprometidos.

La congruencia de los valores institucionales y la Ética como disciplina, deben guiar estos procesos de forma permanente y transversal. Estos elementos serán esenciales a la hora de evaluar el impacto en la formación profesional en la sociedad y así lograr futuros profesionales comprometidos y responsables tanto con la información que la comunidad les transmite y así como con las acciones contextualizadas ofrecidas.

Es entonces relevante reconocer como esta experiencia narrada, aporta a la realidad social actual a través de la formación de profesionales conscientes, quienes se enfrentarán a esta sociedad dañada por la injusticia y vulneración de derechos. Nuestro desafío surge en formación de la educación superior, el respaldo debe ser la búsqueda de la promoción de la justicia, solidaridad y la equidad social. El escenario educativo nos permite contribuir a esta transformación, a través de práctica educativa problematizadoras y acciones en conjunto con la comunidad.

Ante este desafío, se plantea el Aprendizaje-servicio como herramienta metodológica que nos permite acercarnos a la comunidad, generando acciones y proyecciones sociales, desde los contextos universitarios. Es así como los estudiantes, fuera de las aulas, son protagonistas y junto a la comunidad son responsables de importantes cambios sociales. Los estudiantes desarrollan y ponen en prácticas valores de compromiso y ciudadanía, reconociéndose e identificándose como promotores de los derechos humanos y de un cambio social.

\section{RefLeXiOnes finAles}

Se ha logrado reconocer, a través de estas experiencias académicas, que en la metodología de Aprendizaje- servicio los estudiantes se comprometen con una comunidad que presenta necesidades ocupacionales, inician un proceso de vinculación y alianza, que a lo largo de un semestre académico, se traduce en el resultado de un proceso de intervención pertinente y de calidad. En este proceso los estudiantes son protagonistas, guiado por un docente, abordan las necesidades y problemáticas definidas por la comunidad, con un servicio construido con la comunidad, respetando sus valores, cultura e historicidad. De esta forma, tal como propone Freire (1974): "Nadie educa a nadie -nadie se educa a sí mismo- los hombres se educan entre sí mediatizados por el mundo"

El aprendizaje-servicio (AS) rompe con la educación tradicional, la cual se ha caracterizado por un docente protagonista en el aula y un aprendizaje alejado de la realidad.AS responde a las demandas de la sociedad y al desarrollo educativo-profesional, promoviendo la formación de ciudadanos competentes para comprender nuestro mundo, capaces de contribuir y aporta a su desarrollo.

La aplicación de la metodología en la escuela de Terapia Ocupacional, ha sido reconocida a nivel institucional, sin embargo aún existe reticencia por parte de algunas Escuelas, debido a que se requieren cambios estructurales de las asignaturas y sus programas de estudio.

Desde el año 2013 la Universidad Central de Chile, a través del Programa de Desarrollo Social, ha incentivado a la Escuela de Terapia Ocupacional a compartir el éxito de sus experiencias y sus reflexiones, a través de seminarios y encuentros académicos, instancias en las que numerosos docentes, motivados por la metodología, han participado de instancias de capacitación y perfeccionamiento, convencidos del impacto de la metodología, para la formación profesional.

Se destaca también la opinión de los estudiantes que han participado de la asignatura, desarrollando proyectos de servicio, ellos han reconocido que se vive un real proceso de aprendizaje y que requieren un fuerte compromiso y participación activa con la comunidad.

Por otra parte los socios comunitarios, beneficiarios activos de los procesos de acción, destacan y reconocen la experiencia, dentro de las impresiones que se pueden destacar de las evaluaciones en conjunto con socios comunitarios esta el reconocimiento por parte de ellos de articular a la comunidad con el ámbito universitario, destacando el aporte que la comunidad entrega a la formación de futuros Terapeutas Ocupacionales. Es aquí donde tiene sentido citar a Elie Wiesel (citado por Blanco \& Rodríguez, 2012) quien menciona que "ante las atrocidades tenemos que tomar partido. La posición neutral ayuda siempre al opresor, nunca a la víctima. 
El silencio estimula al verdugo nunca al que sufre". No cabe duda entonces, que los terapeutas ocupacionales debemos tomar partido por los cambios sociales y la vulneración de derechos.

En nuestro país, la Educación Superior presenta diversos hitos históricos que denotan cambios sociopolíticos que pretenden dar respuesta a una serie de necesidades en términos de formación de pregrado, es por ello que en cuanto a los desafíos asumidos, se debe seguir promoviendo el uso de esta y otras metodologías que permitan instalar competencias metacognitivas y valores que permitan a los futuros profesionales cumplir un rol precursor en los procesos de desarrollo social. La continuidad de la formación docente y la sistematización de las experiencias de aprendizaje-servicio es un recurso que fortalecerá el proyecto de Escuela y que contribuirá a la difusión necesaria para contribuir a la incorporación de la metodología en otras Escuelas, asignaturas y en diferentes niveles, siendo un reto para los docentes y coordinadores, requiriendo para la validación de la metodología y motivación docente.

Para finalizar, es importante destacar que el interés por sistematizar y difundir esta experiencia, trasciende a compartir un metodología de educación, ya que el énfasis esta puesto en la necesidad y en el desafío de transformación de la educación superior en pro de la formación de futuros profesionales con compromiso y consciencia social, capaces de generar respuestas satisfactorias frente a los fuertes desafíos socioculturales actuales. Debido a esta discusión es que numerosos Terapeutas Ocupacionales han comenzado a reflexionar y mirar de forma más crítica sus acciones profesionales, generando nuevas inquietudes y desafíos, tanto para aquellos en proceso de formación, como para Académicos que comparten un interés en a aportar a cambios en los procesos de Terapia Ocupacional en la comunidad. Cella \& Polinelli (2008) asegura que "es necesario integrarse en los proyectos y acciones que la propia comunidad se encuentra desarrollando con el fin de realizar aportes verdaderamente significativos".

\section{REFERENCIAS BIBLIOGRÁFICAS}

Arratia, A. (2008). Ética, solidaridad y "Aprendizaje Servicio" en la Educación Superior. Acta bioethica, 14, 61-67. Recuperado de http://www.scielo.cl/scielo.php?script=sci_arttext\&pid=S1726$569 \times 2008000100008 \& \operatorname{lng}=\mathrm{es} \& \operatorname{lng}=\mathrm{es}$. $10.4067 / S 1726-$ 569X2008000100008.

Blanco, G., Rodríguez. V (2012) Cambios Sociales y Terapia Ocupacional. Rol del Terapeuta Ocupacional en el Contexto contemporáneo. Revista TOG (A Coruña) 9,190-204. Recuperado de http:// www.revistatog.com/mono/num5/contemporaneo.pdf

Cella, A., Polinelli, S. (2008) Nuevos desafíos en Terapia Ocupacional Comunitaria. Revista Terapia- Ocupacional. Recuperado de http:// www.terapia-cupacional.com/articulos/Nuevos_desafios_Terapia_ocupacional_comunitaria.shtml

Freire, P (1973) Pedagogía del oprimido, Buenos Aires: Siglo XXI

Galheigo, S.M. (2012) Perspectiva crítica y Compleja de Terapia Ocupacional: Actividad, Cotidiano, Diversidad, Justicia Social y Compromiso Ético Político. Revista TOG (A Coruña) 5,176-189.Recuperado de http://www.revistatog.com/mono/num5/compromiso.pdf

Kielhofner, G., (2004) Modelo de Ocupación Humana: “Teoría y Aplicación".Buenos Aires: Ed. Panamericana

Lombardi, G., Rangel, A. (2012) Ciudad educadora, formación y praxis desde el Servicio Comunitario. Serendipia, Revista Electrónica del Programa de Cooperación Interfacultad.1, 42-69. Recuperado de file:///C:/Users/Victor/Downloads/1850-3738-1-SM.pdf

Tapia, M. (2010). Aprendizaje y servicio solidario en el sistema educativo y las organizaciones juveniles. Buenos Aires: Ciudad Nueva.

Torio, S.S., Peña, J.V., Hernández, J. (2014) Aprendizaje Servicio como entrenamiento al emprendimiento social: una experiencia universitaria. Procedia - Social and Behavioral Sciences.139,504-511. Recuperado de http://www.sciencedirect.com/science/article/pii/ S1877042814047090

Torres, N. (2004) Inserción de ejes transversales en la educación superior: visión de los académicos Recuperado de. http://cbtorres. pressbooks.com/chapter/conclusiones/ 